\title{
Qualidade de vida após revascularização do miocárdio:
avaliação segundo duas perspectivas metodológicas*
}

\author{
Quality of life after myocardial revascularization: evaluation according to two methodological perspectives
}

Calidad de vida después de revascularización del miocardio: Evaluación según dos perspectivas metodológicas

\author{
Rosana Aparecida Spadoti Dantas ${ }^{1}$, Lídia Aparecida Rossi ${ }^{1}$, Maria Cristina Silva \\ Costa $^{2}$, Vanessa da Silva Carvalho Vila
}

\begin{abstract}
RESUMO
Objetivo: Analisar a qualidade de vida (QV) segundo a proposta de Flanagan, em dois grupos de pacientes revascularizados; comparar e combinar resultados desta pesquisa com dados de uma etnografia que interpreta significados de QV de pacientes revascularizados. Método: Foi utilizada a triangulação metodológica de duas pesquisas: uma qualitativa (etnográfica) e outra quantitativa, que aplicou a Escala de Qualidade de vida de Flanagan em 124 indivíduos. Resultados: no estudo quantitativo, a QV relacionou-se a "ter e criar filhos" e "relacionamento com os amigos" e no qualitativo a boa QV relacionou-se a bem-estar, felicidade, satisfação, possibilidades na vida e os temas identificados foram: saúde (física, emocional e espiritual), trabalho e harmonia familiar. Conclusão: As dimensões de qualidade de vida identificadas no estudo etnográfico são semelhantes aos domínios que compõem a Escala de Qualidade de vida de Flanagan. A espiritualidade/ religiosidade, não abordada na escala, é destacada pelos participantes como uma dimensão de QV.
\end{abstract}

Descritores: Qualidade de Vida; Antropologia; cultura; Revascularização miocárdica; Avaliação

\begin{abstract}
Objective: To analyze the quality of life (QL) as proposed by Flanagan, in two groups of revascularized patients; to compare and combine results from this research with data from an ethnography study that interprets the meanings that revascularized patients gave to QL. Method: The methodological triangulation was used in two researches: one qualitative (ethnographic) and the other quantitative; the scale of Flanagan was applied in 124 individuals. Results: In the quantitative study the QL was related to "bear and raise children" and "relationships with friends"; in the qualitative study a good QL was related to wellbeing, happiness, satisfaction, opportunities in life. The identified issues were: health (physical, emotional and spiritual), work and family harmony. Conclusion: The dimensions of quality of life identified in the ethnographic study were similar to the domains of Flanagan's QL-Scale. The spirituality/religiosity, not contemplated in the scale, is highlighted by participants as an aspect of the QL.
\end{abstract}

Keywords: Quality of life; Anthropology; Culture; Myocardial revascularization; Evaluation

\section{RESUMEN}

Objetivo: Analizar la calidad de vida (CV) según la propuesta de Flanagan, en dos grupos de pacientes revascularizados; comparar y combinar resultados de esta investigación con datos de una etnografía que interpreta significados de CV de pacientes revascularizados. Método: Fue utilizada la triangulación metodológica de dos investigaciones: una cualitativa (etnográfica) y otra cuantitativa, que aplicó la Escala de Calidad de vida de Flanagan en 124 individuos. Resultados: en el estudio cuantitativo, la CV se relacionó a "tener y criar hijos" y al "relacionamiento con los amigos" y en el cualitativo la buena CV se relacionó al bienestar, felicidad, satisfacción, posibilidades en la vida y los temas identificados fueron: salud (física, emocional y espiritual), trabajo y harmonía familiar. Conclusión: Las dimensiones de calidad de vida identificadas en el estudio etnográfico son semejantes a los dominios que componen la Escala de Calidad de vida de Flanagan. La espiritualidad/religiosidad, no abordada en la escala, es destacada por los participantes como una dimensión de la CV.

Descriptores: Calidad de vida; Antropología; Cultura; Revascularización miocárdica; Evaluación

\footnotetext{
* Trabalhos realizados no Hospital das Clínicas de Ribeirão Preto - Ribeirão Preto (SP), e, em um hospital de grande porte de Goiânia (GO), Brasil.

1 Professora Associada do Departamento de Enfermagem Geral e Especializada da Escola de Enfermagem de Ribeirão Preto da Universidade de São Paulo - USP. Ribeirão Preto (SP), Brasil.

${ }^{2}$ Professora Doutora do Departamento de Enfermagem Psiquiátrica e Ciências Humanas da Escola de Enfermagem de Ribeirão Preto da Universidade de São Paulo - USP.

${ }^{3}$ Professora Assistente I do Departamento de Enfermagem, Nutrição e Fisioterapia da Universidade Católica de Goiás - Goiânia (GO), Brasil.
} 


\section{INTRODUÇÃO}

A qualidade de vida relacionada à doença arterial coronariana, o impacto do tratamento na vida dos indivíduos constitui, atualmente, objeto de investigação para diferentes pesquisadores, com distintas abordagens ${ }^{(1-5)}$.

Pesquisas qualitativas destacam os significados de qualidade de vida elaborados por pacientes cardíacos, e consideram o fato de que significados específicos são coletivamente produzidos, configurando-se como construções sócio-culturais que se refletem nas interpretações dos indivíduos e são reinterpretadas por eles ${ }^{(())}$. Mais freqüentes na investigação sobre qualidade de vida relacionada com cardiopatia, pesquisas quantitativas mensuram-na por meio da aplicação de escalas.

Outras investigações recorrem, ainda, à triangulação metodológica ${ }^{(7-8)}$ definida como a combinação de várias fontes de dados - triangulação múltipla - ou abordagens metodológicas ${ }^{(9)}$. A triangulação metodológica pode ser classificada como simultânea, com os métodos qualitativos e quantitativos empregados ao mesmo tempo, e seqüencial, quando os resultados de um método são essenciais para planejar os passos do próximo método ${ }^{(10)}$. Além da triangulação metodológica, outros tipos têm sido definidos: triangulação entre pesquisadores, teórica e analítica ${ }^{(11)}$. As situações em que se recorre à triangulação são: primeiro, quando um único método é insuficiente para uma abordagem que permita capturar a abrangência de um fenômeno ${ }^{(7)}$; segundo, para assegurar a validação de instrumentos ou de conceito (validade

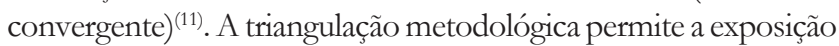
de informações que poderiam permanecer desconhecidas com o uso de um único método ${ }^{(12)}$, bem como assegura, pela interpenetração de métodos, a possibilidade de interpretação mais abrangente.

O problema de pesquisa aqui exposto tem sido enfocado, predominantemente, do ponto de vista quantitativo. Instrumentos para mensurar qualidade de vida, propostos para populações específicas, são adaptados e utilizados para populações de outros países, que vivem em contexto cultural diferente, o que tem gerado questionamentos.

Reconhecendo que a combinação de estudos construídos com diferentes métodos pode trazer maior conhecimento sobre qualidade de vida associada à cirurgia de revascularização do miocárdio, o presente estudo teve como objetivos: analisar a qualidade de vida (QV), segundo a proposta de Flanagan, em dois grupos de pacientes revascularizados; comparar e combinar resultados desta pesquisa com dados de uma etnografia que interpreta significados de QV de pacientes revascularizados.

\section{MÉTODOS}

Este artigo utiliza a triangulação de dados provenientes de dois estudos realizados por investigadores de um mesmo grupo de pesquisa, que abordaram a qualidade de vida de indivíduos coronariopatas após tratamento cirúrgico. Foram aprovados pelos comitês de ética das respectivas instituições nas quais foram realizados, tendo os participantes sua identidade protegida por anonimato e assinado o Termo de Consentimento Livre e
Esclarecido.

Um dos estudos, caracterizado como quantitativo, descritivo e observacional, teve como objetivo avaliar a qualidade de vida de 124 pacientes submetidos à cirurgia de revascularização do miocárdio há, no mínimo, seis meses e em seguimento ambulatorial no Hospital das Clínicas de Ribeirão Preto/SP (HCRP). Adotou-se a definição de qualidade de vida proposta por Flanagan ${ }^{(13)}$ e relacionada à satisfação dos indivíduos com o bem-estar físico e material; relações com outras pessoas; envolvimento em atividades sociais, comunitárias e cívicas; desenvolvimento, enriquecimento pessoal e recreação. Para a avaliação da qualidade de vida nesse estudo, foi utilizada a escala elaborada por Flanagan ${ }^{(13)}$, em sua versão adaptada por Burckhardt ${ }^{(14)}$ e com a inclusão do item independência. Os 16 itens são respondidos usando uma escala de sete pontos, que varia de um (extremamente insatisfeito) até sete (extremamente satisfeito). A avaliação da qualidade de vida é obtida pela soma dos valores das respostas aos itens (intervalo possível entre $16 \mathrm{e}$ 112 pontos), com uma alta pontuação indicando elevada satisfação com os domínios avaliados. Os dados, coletados por entrevistas individuais com os pacientes foram, posteriormente, processados e analisados usando o programa de software Statistical Package for Social Science (SPSS) versão 15.0. Realizaramse análises descritivas, com medida de posição (média) e de variabilidade (desvio-padrão). Maiores detalhes dessa investigação já se encontram publicados ${ }^{(15-16)}$.

A outra pesquisa, etnográfica ${ }^{(17)}$, propôs a interpretação dos significados elaborados a partir da experiência da doença e da cirurgia, inserindo-os em seu contexto cultural ${ }^{(18)}$. O estudo orientou-se teoricamente pela antropologia interpretativa, cujo principal expoente, Clifford Geertz, concebe a etnografia como uma "descrição densa", que busca a interpretação de significados tendo discursos e comportamentos manifestos como dados empíricos $^{(18)}$. O grupo pesquisado foi composto por 11 indivíduos também submetidos ao tratamento cirúrgico da doença arterial coronariana em hospital de grande porte da cidade de Goiânia/GO, com tempo de pós-operatório mínimo de seis meses e máximo de dois anos e em seguimento ambulatorial. A coleta de dados do estudo etnográfico incluiu formulário de identificação sócio-econômica e técnicas características deste tipo de pesquisa: observação direta e entrevista antropológica ${ }^{(19)}$. As entrevistas tiveram roteiro previamente elaborado de tópicos selecionados, foram gravadas e conduzidas como processo dialógico ${ }^{(19)}$. As seguintes questões foram utilizadas para guiar as entrevistas: o que você considera prioritário e importante em sua vida; o que significa ter qualidade de vida para você? Delimitouse o número de entrevistas, em duas a quatro para cada sujeito, pelo critério de saturação dos conteúdos, considerando-se os objetivos da pesquisa. As entrevistas subseqüentes à primeira permitiram esclarecer inconsistências e contradições. Em estudos etnográficos o processo de coleta e análise de dados é concomitante, possibilitando ao investigador esclarecer em encontros subseqüentes aspectos coletados no primeiro, até que não existam mais fatos novos, ou seja, tenha ocorrido a saturação dos dados.

A análise interpretativa exigiu a leitura repetida de entrevistas e registros de observação, assegurando a "impregnação dos dados 
pelo pesquisador ${ }^{\text {(19) }}$. Os dados, sistematizados progressivamente pela procura de regularidades e diferenças nos sentidos expressados pelos sujeitos, convergiram para unidades de significados. Unidades semelhantes originaram os temas, cuja análise envolveu a busca de padrões, regras e valores subjacentes aos sentidos atribuídos pelos sujeitos a suas experiências, para desvendar a lógica cultural em que se orientam ${ }^{(18,20)}$. Após a coleta dos dados etnográficos, os participantes também responderam à Escala de Qualidade de Vida de Flanagan (EQVF) para comparação com os dados do estudo de Ribeirão Preto.

Após a análise e interpretação dos resultados de cada estudo, respeitando-se as duas abordagens, quantitativa e qualitativa, procurou-se combinar os resultados de forma integrada, buscando tanto dados concordantes como discordantes. Combinar dados não ocorre no processo de análise, mas na comparação dos resultados de cada estudo de forma coesa e coerente $^{(10)}$. A seguir, são apresentados os resultados dos dois estudos e, no tópico de discussão, a combinação dos resultados. Para a realização do presente artigo também foram usados os bancos de dados originais dos estudos supracitados.

\section{RESULTADOS}

Entre os 124 participantes do HCRP, a maioria era do sexo masculino $(77,62, \%)$, aposentados ou sem desempenho de atividades remuneradas $(90,73 \%)$ e com idades variando entre 38,7 e 80 anos (média de 62 anos). No grupo de pacientes investigados em Goiânia, das 11 pessoas submetidas à cirurgia, sete eram homens e quatro mulheres, todos aposentados ou sem desempenho de atividades remuneradas após a cirurgia e com idades entre 53 e 74 anos (média de 63 anos) idem. No grupo de Ribeirão Preto, o tempo médio de cirurgia cardíaca foi de 31,2 meses (intervalo de seis meses a 6,6 anos) enquanto no grupo de Goiânia, esse tempo foi menor (média de 11,5 meses idem, intervalo de seis meses a um ano e nove meses).

No que se refere aos resultados obtidos com a Escala de Qualidade de Vida de Flanagan (EQVF), entre os participantes de Ribeirão Preto, obtivemos como resposta, valores entre 44 e $112(\bar{x}=84,79$ e $s=13,54)$, sendo que as respostas aos itens variaram de 2,75 a $7(=5,3$ e $s=0,8)$. Ambos os resultados, soma total dos itens da escala e média dos itens, refletem alto grau de satisfação com os aspectos abordados pelo instrumento, estando os participantes entre satisfeitos e bastante satisfeitos com os domínios investigados da qualidade vida. Os itens levantados como fontes de maior satisfação entre os participantes foram: ter e criar filhos $(\bar{x}=6,0$ e $s=1,3)$ e relacionamento com os amigos $(\bar{x}=5,7$ e $s=1,4)$. Os itens de menor satisfação foram: participar de atividades recreacionais e esportivas $(\bar{x}=4,6$ e s=1,6) e saúde (sentir-se fisicamente bem e vigoroso) $(\bar{x}=4,8$ e $s=1,5)$.

Tabela 1. Participantes, segundo freqüência de respostas fornecidas para o grau de satisfação com os itens da EQVF.

\begin{tabular}{|c|c|c|c|c|c|}
\hline \multirow[t]{2}{*}{ Domínios da EQVF } & \multirow[t]{2}{*}{ Itens } & \multicolumn{2}{|c|}{$\begin{array}{l}\text { Insatisfeitos e nem } \\
\text { satisfeito/nem insatisfeito }\end{array}$} & \multicolumn{2}{|c|}{ Satisfeitos } \\
\hline & & $\begin{array}{c}\text { Rib eirão } \\
\text { Preto }\left(n^{\circ} \%\right)\end{array}$ & $\begin{array}{l}\text { Goiânia } \\
\text { n }^{\text {o } *}\end{array}$ & $\begin{array}{l}\text { Rib eirão } \\
\text { Preto }\left(\mathrm{n}^{\mathbf{0}} \%\right)\end{array}$ & $\begin{array}{l}\text { Goiânia } \\
\text { n }^{\circ} *\end{array}$ \\
\hline \multirow[t]{2}{*}{$\begin{array}{l}\text { Bem-estar físico e } \\
\text { material }\end{array}$} & $\begin{array}{l}1 \text { - Conforto material: moradia, alimentação, } \\
\text { situação financeira }\end{array}$ & $35(28,2 \%)$ & 3 & $89(71,8 \%)$ & 7 \\
\hline & $\begin{array}{l}2 \text { - Saúde: sentir-se fisicamente bem e cheio de } \\
\text { energia }\end{array}$ & $39(31,5 \%)$ & 3 & $85(68,5 \%)$ & 7 \\
\hline \multirow[t]{4}{*}{$\begin{array}{l}\text { Relações com outras } \\
\text { pessoas }\end{array}$} & $\begin{array}{l}3 \text { - Relacionamento com pais, irmãos e outros } \\
\text { parentes: conviver e ajudar }\end{array}$ & $22(17,7 \%)$ & 1 & $102(82,3 \%)$ & 9 \\
\hline & 4 - Ter e criar filhos & $18(14,5 \%)$ & 1 & $106(85,5 \%)$ & 9 \\
\hline & 5 - Relacionamento com cônjuge ou parceiro(a) & $35(28,2 \%)$ & 3 & $89(71,8 \%)$ & 7 \\
\hline & 6 - Relacionamento com amigos & $17(13,7 \%)$ & 1 & $107(86,3 \%)$ & 9 \\
\hline \multirow{2}{*}{$\begin{array}{l}\text { Envolvimento em } \\
\text { atividades sociais, } \\
\text { comunitárias e cívicas }\end{array}$} & 7 - Ajudar e apoiar outras pessoas & $19(15,3 \%)$ & 2 & $105(84,7 \%)$ & 8 \\
\hline & $\begin{array}{l}8 \text { - Participação em as sociações comunitárias e } \\
\text { atividades de interesse público }\end{array}$ & $39(31,5 \%)$ & 8 & $85(68,5 \%)$ & 2 \\
\hline \multirow{4}{*}{$\begin{array}{l}\text { Desenvolvimento e } \\
\text { enriquecimento } \\
\text { pessoal }\end{array}$} & $\begin{array}{l}9 \text { - Aprendizado: poder aumentar seus } \\
\text { conhecimentos gerais }\end{array}$ & $37(29,8 \%)$ & 5 & $87(70,2 \%)$ & 5 \\
\hline & $\begin{array}{l}10 \text { - Autoconhecimento: saber sobre suas forças e } \\
\text { suas limitações, saber aonde quer chegar e quais os } \\
\text { objetivos importantes para a sua vida. }\end{array}$ & $34(27,4 \%)$ & 4 & $90(72,6 \%)$ & 6 \\
\hline & 11 - Trabalho no emprego ou em casa & $32(25,8 \%)$ & 3 & $92(74,2 \%)$ & 7 \\
\hline & 12 - Conseguir se comunicar & $23(18,5 \%)$ & 1 & $101(81,5 \%)$ & 9 \\
\hline \multirow[t]{3}{*}{ Recreação } & $\begin{array}{l}13 \text { - Participar de atividades de recreação e } \\
\text { esportivas }\end{array}$ & $56(45,2 \%)$ & 7 & $68(54,8 \%)$ & 3 \\
\hline & 14 - Ouvir música, ler, assistir TV ou ir ao cinema & $20(16,9 \%)$ & 1 & $104(83,1 \%)$ & 9 \\
\hline & $\begin{array}{l}15 \text { - Encontrar-se com outras pessoas e fazer coisas } \\
\text { juntos }\end{array}$ & $23(18,5 \%)$ & 2 & $101(81,5 \%)$ & 8 \\
\hline Independência & 16 - Sentir-se capaz de fazer as cois as por si mes mo & $24(19,4 \%)$ & 5 & $100(80,6 \%)$ & 5 \\
\hline
\end{tabular}


Entre os 11 participantes de Goiânia, sujeitos também da pesquisa etnográfica, um não completou a EQVF. Nesse grupo, o valor total da soma das respostas aos itens variou de 65 e 96 $(\bar{x}=78,4$ e $s=8,7)$ e as respostas aos itens variaram de 4,1 a $6(\bar{x}=4,9$ e $s=0,54)$, mostrando que eles encontravam-se entre nem insatisfeito, nem satisfeito e satisfeito com os aspectos abordados pelo instrumento. Os itens com maiores médias, indicando maior grau de satisfação para o grupo foram: ter e criar filhos $(\bar{x}=5,7 \mathrm{e}$ $s=1,0)$ e relacionamento com os amigos $(\bar{x}=5,6$ e $s=1,0)$. Os de menor satisfação foram: participar de atividades recreacionais e esportivas $(\bar{x}=3,8$ e $s=0,9)$ e aprendizado $(\bar{x}=4,2$ e $s=1,2)$.

A análise dos dados provenientes do total de 124 pacientes demonstrou que ter e criar filhos e relacionamento com os amigos foram os itens apontados com maior satisfação para eles. No que se refere à satisfação com o item saúde: sentir-se fisicamente bem e vigoroso, a mesma média das respostas foi obtida nos dois grupos, embora, quando comparada à satisfação do grupo de Goiânia aos demais itens da escala, constatou-se que eles se apresentaram menos satisfeitos com outros aspectos de suas vidas do que com a sua condição de saúde.

Posteriormente, os sujeitos foram agrupados em dois conjuntos. No primeiro grupo, colocaram-se os pacientes que responderam, em cada um dos itens da escala de Flanagan, as opções: extremamente insatisfeito, muito insatisfeito, insatisfeito e nem insatisfeito/nem satisfeito e no segundo grupo, inseriramse aqueles que responderam estar satisfeito, muito satisfeito e extremamente satisfeito. Os resultados dos participantes de Ribeirão Preto e de Goiânia estão na tabela 1.

Os dados demonstraram satisfação de mais da metade dos participantes de Ribeirão Preto com os itens investigados e que compõem os domínios da qualidade de vida no referencial de Flanagan. O item que apresentou menor número de participantes satisfeitos foi Participar de atividades de recreação e esportivas (68; 54,8\%). Em seguida, 85 (68,5\%) sujeitos optaram igualmente pelas respostas que representavam sua satisfação diante dos itens: Saúde: sentir-se fisicamente bem e cheio de energia e Participação em associações comunitárias e atividades de interesse público. Os itens Relacionamento com amigos e Ter e criar filhos foram aqueles com maior freqüência de respostas para as opções de satisfação, com $107(86,3 \%)$ e 106 (85,5\%) sujeitos, respectivamente. Resultados similares foram obtidos no grupo de Goiânia para os itens com menor freqüência de participantes satisfeitos: Participar de atividades de recreação e esportivas, com três sujeitos, e Participação em associações comunitárias e atividades de interesse público, com apenas dois. Nove sujeitos desse grupo apresentaram algum grau de satisfação com três itens do domínio "Relações com outras pessoas" e com os itens Ouvir música, ler, assistir TV ou ir ao cinema e Conseguir se comunicar.

Com relação aos dados obtidos pela pesquisa etnográfica, voltada para interpretar significados da qualidade de vida dos 11 participantes, constatou-se que a vida de boa qualidade relacionava-se com bem-estar, felicidade, satisfação, possibilidades na vida. Os temas identificados foram: saúde, trabalho e harmonia familiar. O primeiro tema (expressado como "ter saúde") inclui os aspectos relacionados à saúde física ("alimentar-se bem", "dormir", "não sentir dor e fraqueza", "tomar remédio"), saúde emocional ("namorar", "dançar", "ter paz e tranqüilidade na cabeça") e saúde espiritual ("ter fé", "acreditar em Deus”). A saúde, para os sujeitos, é o indicador "mais importante" de qualidade de vida, essencial para que possam "trabalhar, ganhar dinheiro e cuidar da família”. Por um lado, os significados atribuídos à saúde convergem para avaliações normativas: [...] saúde, você não sentir nada! Estar tranqüilo com a vida boa [trabalho e dinheiro], sentindo dor nenhuma! (S3, M, 56). [...] é você comer bem, alimentar bem, dormir bem, rezar para Deus dá força. [...] inclusive até namoro também! (S9, M, 62). [...] saúde é ter fé em Deus! (S4, F, 53). Por outro lado, as apreciações sobre a própria saúde refletem ambigüidades: [...] sou doente, mas me sinto saudável! Sou cheia de problema de saúde, mas não me entrego a ela, tenho fé em Deus e paz na minha família, então tenho saúde, não é, porque não é só o físico é a mente também! (S1, F, 74).

A capacidade para trabalhar foi identificada pelos sujeitos como sinal de saúde e qualidade de vida, sendo o trabalho significado como fonte de recursos financeiros e visto como garantia de aquisição de bens e promoção do bem-estar material da família. Todos expressaram insatisfação relativa a trabalho, afirmando experimentar limitações para retomar suas atividades, após a cirurgia, o que repercute em percepção de baixa auto-estima e de dependência financeira.

A harmonia familiar, tida como condição essencial para a qualidade de vida, indica a atribuição à família do significado de núcleo importante de produção de sentidos e de apoio a seus membros, tanto financeiro quanto emocional. Outro núcleo importante de sentidos e apoio ressalta em expressões que permearam as narrativas dos sujeitos, tanto nos relatos sobre a experiência da doença como sobre significados de qualidade de vida: entregar a vida nas mãos de Deus, acreditar que Deus dá força, Deus sabe o que faz, não reclamar porque Deus quis assim, ter fé traz conforto e esperança. Esse núcleo foi mencionado como uma importante condição para ter saúde.

De modo geral, ainda que os participantes tenham declarado não estar satisfeitos com todas as dimensões que originaram os temas, afirmaram-se felizes. Avaliaram positivamente sua qualidade de vida, apesar de não se considerarem sadios e de enfrentarem problemas sócio-econômicos decorrentes das limitações impostas pelas condições de saúde (desemprego, aposentadoria por invalidez, dependência de cuidados familiares), como pode ser observado a seguir: [...] porque eu não tenho saúde, mas Deus dá um jeito! Não pode reclamar não porque graças a Deus nós ainda estamos andando, não estamos dependendo de favor dos outros, vivendo em cadeira de rodas, em cima de uma cama, temos casa, comida! (S5, M, 65); [...] a pessoa estar alegre, valorizar as coisas que tem que Deus dá para gente! [...] eu tenho que me colocar no meu lugar que sou pobre! Então se conformar com tudo que Deus dá para a gente; não se queixar da sorte, que é infeliz... (S1, F, 74); [...] tenho uma boa qualidade de vida, mas ao mesmo tempo não tenho, pois falta servico, falta dinheiro, falta o que comer, mas não pode reclamar não! (S3, M, 56).

\section{DISCUSSÃO}

No que se refere à avaliação da qualidade de vida por meio da aplicação da EQVF, existem similaridades entre os participantes de Ribeirão Preto e de Goiânia. Observou-se uma medida que 
reflete boa avaliação da qualidade de suas vidas, após terem sido submetidos à revascularização cirúrgica do miocárdio, sendo os valores obtidos semelhantes aos encontrados no estudo que originou o instrumento ${ }^{(13)} \mathrm{e} \mathrm{em}$ outros estudos com indivíduos cardiopatas $^{(2,21)}$. Entre nossos participantes, os itens de maior satisfação foram Ter e criar filhos e Relacionamento com amigos, o que diferiu de pacientes norte-americanos revascularizados, os quais indicaram maior satisfação para os itens Independência e Relacionamento com cônjuge/pessoa significante ${ }^{(2)}$. Entretanto, a comparação com outras pesquisas brasileiras permitiu a constatação de que Ter e criar filhos também foi um dos itens apontados como os de maior satisfação para idosos freqüentadores de uma universidade da terceira idade ${ }^{(22)}$ e para idosos nordestinos ${ }^{(23)}$.

Entre os participantes de Ribeirão Preto, os itens que receberam menores valores para a satisfação, na aplicação da escala, foram: Participar de atividades recreacionais e esportivas e Saúde (sentir-se bem e cheio de vida) e, entre os participantes goianos, foram Participar de atividades recreacionais e esportivas e Aprendizado. Considerando-se a presença de vários idosos entre os participantes, percebe-se grande diferença na comparação desses resultados com os de outros estudos realizados entre idosos, no Brasil, com o mesmo instrumento de avaliação: entre os idosos freqüentadores da universidade aberta à terceira idade, o item Recreação foi um dos mais satisfatórios ${ }^{(22)}$. Nessa amostra de idosos, a média do item Saúde também foi maior $(=5,09 ; \mathrm{s}=1,30)$, o que reflete a disposição dos mesmos para participarem de cursos para terceira idade, enquanto a amostra do estudo aqui focalizado foi obtida de uma população de pacientes cardiopatas, com atendimento ambulatorial em hospital universitário. Já entre os idosos do Nordeste, o item Saúde teve média inferior às obtidas nos estudos que baseiam este $\operatorname{artigo}^{(23)}$.

Como as respostas aos itens do instrumento de qualidade de vida podem ser fornecidas em uma escala de sete pontos que varia de um (extremamente insatisfeito) até sete (extremamente satisfeito), constatou-se que, para os participantes de Ribeirão Preto e de Goiânia, a satisfação com o item Saúde variou de "nem satisfeito, nem insatisfeito" a "satisfeito". Tal resultado também havia sido encontrado entre sobreviventes de uma parada cardiorespiratória ${ }^{(21)}$ e entre pacientes revascularizados americanos $^{(2)}$.

A pesquisa etnográfica realizada com os participantes de Goiânia indica que, para eles, uma vida de boa qualidade tem seus significados subordinados à saúde, trabalho e harmonia familiar. Se isto se revela problemático, em função das limitações decorrentes da doença e cirurgia, não representa, entretanto, empecilho intransponível para a satisfação com a qualidade de vida. Os sujeitos revelaram, entretanto, insatisfação em suas avaliações de qualidade de vida subordinadas ao trabalho; ao mesmo tempo, impedidos de trabalhar dadas as seqüelas da doença, concebem-se como felizes. Dos resultados da pesquisa etnográfica, depreendem-se dois núcleos de significação que se manifestam concomitantemente: o apoio moral da satisfação com a qualidade de vida, expresso na valorização da família e na forte vinculação com o sagrado como fontes de significados para suas experiências, aparece juntamente com a racionalidade econômica presidindo a associação entre qualidade de vida e trabalho. Todavia, a comparação dos resultados da pesquisa etnográfica com aqueles obtidos pela aplicação da escala de qualidade de vida permite constatar discrepâncias: sete entre os dez sujeitos investigados em Goiânia responderam a opção "satisfeito" para os itens Trabalho e Saúde; no entanto, a investigação etnográfica revela que, quanto à saúde, "resignação" seria a definição mais adequada e, quanto ao trabalho, a incapacidade para realizá-lo é a sua principal fonte de insatisfação. Neste caso, a pesquisa etnográfica parece ter assegurado ultrapassar uma possível tendência a não manifestar insatisfações diante do que a vida lhes apresenta. Contradições permeiam a comparação dos resultados da pesquisa etnográfica com os da aplicação da escala, quando se analisa a satisfação com o trabalho. Enquanto o item da escala "Trabalho no emprego ou em casa" é avaliado como "satisfatório" por $70 \%$ ou mais dos sujeitos dos dois grupos, os participantes de Goiânia, na abordagem etnográfica, revelaram suas insatisfações decorrentes da incapacidade para trabalhar e esclareceram a subordinação da qualidade de vida ao trabalho. Possivelmente, a focalização da satisfação com o trabalho, pela escala, tenha se revelado insuficiente para apreender a importância dele na vida dessas pessoas.

\section{CONCLUSÃO}

No processo de triangulação metodológica dos resultados dos dois estudos, chama a atenção o fato de que dimensões de qualidade de vida mencionadas pelos sujeitos no estudo etnográfico são semelhantes aos domínios que compõem a EQVF. Apesar de esta e de outras semelhanças, como relativa satisfação com a saúde, na aplicação da escala, ou aceitação das condições de saúde, na pesquisa etnográfica, e a importância da família para a satisfação com a qualidade de vida, nos dois estudos, os resultados também contêm discrepâncias. A espiritualidade/ religiosidade, identificada no estudo etnográfico como um aspecto importante relacionado a ter saúde, não é abordada na EQVF.

A satisfação com a qualidade de vida, verificada nos resultados da aplicação da escala e identificada de maneira parcial na pesquisa etnográfica, manifesta-se contraditoriamente, quando associada ao trabalho. Os resultados da pesquisa etnográfica indicam, como principal fonte de atribuição de significados negativos à qualidade de vida, o impedimento de trabalhar e a dependência financeira decorrente da doença, fatos não verificados com a aplicação da escala.

Embora este estudo não possibilite generalizações, os resultados chamam atenção para o fato de que é importante utilizar delineamentos metodológicos que permitam captar as reais perspectivas dos sujeitos quando buscamos compreender as dimensões de qualidade de vida.

\section{AGRADECIMENTOS}

Os autores agradecem à FAPESP pelo subsídio recebido para a realização dos estudos analisados neste artigo (Processo FAPESP 02/12360-7). 


\section{REFERÊNCIAS}

1. Carvalho F, Telarolli Júnior R, Machado JCMS. Uma investigação antropológica na terceira idade: concepções sobre a hipertensão arterial. Cad Saúde Pública $=$ Rep Public Health. 1998;14(3):617-21.

2. Dantas RA, Motzer SA, Ciol MA. The relationship between quality of life, sense of coherence and self-esteem in persons after coronary artery bypass graft surgery. Int J Nurs Stud. 2002;39(7):745-55.

3. Cella D, Nowinski CJ. Measuring quality of life in chronic illness: the functional assessment of chronic illness therapy measurement system. Arch Phys Med Rehabil. 2002;83(12 Suppl 2):S10-7.

4. Barnason S, Zimmerman L, Anderson A, Mohr-Burt S, Nieveen $\mathrm{J}$. Functional status outcomes of patients with a coronary artery bypass graft over time. Heart Lung. 2000;29(1):33-46.

5. Spertus JA, Winder JA, Dewhurst TA, Deyo RA, Fihn SD. Monitoring the quality of life in patients with coronary artery disease. Am J Cardiol. 1994;74(12):1240-4.

6. Padilla GV, Kagawa-Singer M. Quality of life and culture. In: King CR, Hinds PS. Quality of life: from nursing and patient perspectives: theory, research, practice. Boston: Jones and Bartlett Publishers; 1998. p.74-92.

7. Lukkarinen H. Methodological triangulation showed the poorest quality of life in the youngest people following treatment of coronary artery disease: a longitudinal study. Int J Nurs Stud. 2005;42(6):619-27.

8. Wyrwich KW, Metz SM, Kroenke K, Tierney WM, Babu AN, Wolinsky FD. Triangulating patient and clinician perspectives on clinically important differences in health-related quality of life among patients with heart disease. Health Serv Res. 2007;42(6 Pt 1):2257-74; discussion 2294-323.

9. Denzin NK, Lincoln YS, editors. Strategies of qualitative inquiry. Thousand Oaks: Sage; 1998.

10. Morse JM. Approaches to qualitative-quantitative methodological triangulation. Nurs Res. 1991;40(2):120-3.
11. Patton MQ. Qualitative research \& evaluation methods. 3rd. ed. Thousand Oaks: Sage; 2002.

12. Thurmond VA. The point of triangulation. J Nurs Scholarsh. 2001;33(3):253-8.

13. Flanagan JC. Measurement of quality of life: current state of the art. Arch Phys Med Rehabil. 1982;63(2):56-9.

14. Burckhardt CS, Woods SL, Schultz AA, Ziebarth DM. Quality of life of adults with chronic illness: a psychometric study. Res Nurs Health. 1989;12(6):347-54

15. Dantas RAS, Góis CFL, Silva LM. Utilização da versão adaptada da escala de qualidade de vida de Flanagan em pacientes cardíacos. Rev Latinoam Enferm. 2005;13(1):15-20.

16. Dantas RAS, Ciol MA. Quality of life after coronary artery bypass surgery. West J Nurs Res. 2008; 30(4):477-90.

17. Vila VSC, Rossi LA. Quality of life from the perspective of revascularized patients during rehabilitation: an ethnographic study . Rev Latinoam Enferm. 2008;16(1):7-14.

18. Geertz C. A interpretação das culturas. Rio de Janeiro: LTC Livros Técnicos Científicos Editora; 1989.

19. Romanelli G, Biasoli-Alves ZMM, organizadores. Diálogos metodológicos sobre prática de pesquisa. Ribeirão Preto: Legis Summa; 1998

20. Miles MB, Huberman AM. Qualitative data analysis: an expanded sourcebook. 2nd. ed. Thousand Oaks: Sage; 1994.

21. Motzer SU, Stewart BJ. Sense of coherence as a predictor of quality of life in persons with coronary heart disease surviving cardiac arrest. Res Nurs Health. 1996;19(4):287-98.

22. Gonçalves LHT, Dias MM, Liz TG. Qualidade de vida de idosos independentes segundo proposta de avaliação de Flanagan. Mundo Saúde (1995). 1999;23(4):214-20.

23. Santos SR, Santos IBC, Fernandes MGM, Henriques MERM. Qualidade de vida do idoso na comunidade: aplicação da Escala de Flanagan. Rev Latinoam Enferm. 2002;10(6):757-64. 